\title{
Hermite Finite Element Method for a Class of Viscoelastic Beam Vibration Problem
}

\author{
Ying Tang, Zhe Yin \\ School of Mathematics and Statistics, Shandong Normal University, Jinan, China \\ Email: ZYin_sdnu@163.com
}

How to cite this paper: Tang, Y. and Yin, Z. (2021) Hermite Finite Element Method for a Class of Viscoelastic Beam Vibration Problem. Engineering, 13, 463-471. https://doi.org/10.4236/eng.2021.138033

Received: July 27, 2021

Accepted: August 17, 2021

Published: August 20, 2021

Copyright $\odot 2021$ by author(s) and Scientific Research Publishing Inc. This work is licensed under the Creative Commons Attribution International License (CC BY 4.0).

http://creativecommons.org/licenses/by/4.0/

\begin{abstract}
Beam equation can describe the deformation of beams and reflect various bending problems and it has been widely used in large engineering projects, bridge construction, aerospace and other fields. It has important engineering practice value and scientific significance for the design of numerical schemes. In this paper, a scheme for vibration equation of viscoelastic beam is developed by using the Hermite finite element. Based on an elliptic projection, the errors of semi-discrete scheme and fully discrete scheme are analyzed respectively, and the optimal $L^{2}$-norm error estimates are obtained. Finally, a numerical example is given to verify the theoretical predictions and the validity of the scheme.
\end{abstract}

\section{Keywords}

Viscoelastic Beam, Hermite Element, Error Estimate, Numerical Simulation

\section{Introduction}

Consider the following viscoelastic beam vibration problem:

$$
\left\{\begin{array}{l}
\frac{\partial^{2}}{\partial x^{2}}\left(E I \frac{\partial^{2} u}{\partial x^{2}}\right)+\rho_{0} S u_{t t}+\mu u_{t}+k u=f(x, t), x \in(0, L), t \in(0, T], \\
u(x, 0)=\varphi(x), u_{t}(x, 0)=\psi(x), x \in[0, L], \\
u(0, t)=u(L, t)=0, u_{x x}(0, t)=u_{x x}(L, t)=0, t \in[0, T],
\end{array}\right.
$$

where $u(x, t)$ represents the transverse displacement of the beam, also known as the deflection, $E I$ is the bending stiffness, $\rho_{0}$ is the density, $S$ is the cross-sectional area, $\mu>0$ is the damping coefficient, $L$ is the constant, $\varphi(x), \psi(x)$ and $f(x, t)$ are smooth functions which are given known.

Beam is the most common component of mechanical equipment and engineering structure. Beam equation can describe the deformation of beams and reflect 
various bending problems. It has been widely used in large engineering projects, bridge construction, aerospace and other fields. Its theoretical and numerical methods have been paid much attention. In [1], the viscoelastic dynamic beam model was studied by using the finite element method and Newmark method. Pierro [2] not only studied the transverse vibration of the viscoelastic beam, but also enlightened the damping characteristics of the structure. Huang, Kouami, et al. [3] [4] presented the finite element scheme for viscoelastic sandwich beam in 2019 and 2020 respectively. Snchez [5] proposed a finite element formulation for thick arbitrarily laminated beams. In [6] the Faedo-Galerkin method was used to solve beam vibration equation with nonlinear tension, a viscoelastic damping and distributed delay term. Wu [7] studied the viscoelastic string-beam coupled system in detail. In [8], the one-dimensional finite element method and analytical method were used to study Euler-Bernoulli beams equations resting on multi-layered viscoelastic soil. A new numerical method was considered in [9] for the equations of fractional-order viscoelastic Euler-Bernoulli beams. Among the aforementioned methods, the finite element method has the characteristics of flexible mesh generation, wide application area and high calculation accuracy. The cubic Hermite element can guarantee the continuity of the first derivative of the interpolation function, and at the same time, the displacement value $u$ and the angle value $u^{\prime}$ can be calculated [10]. However, there is little work about the Hermite finite element method for viscoelastic beam vibration problem. Therefore, in this paper, a finite element calculation scheme is established for problem (1) based on Hermite element. Using elliptic projection operator, the error analysis is carried out and the convergence order of the scheme is proved to be $O\left(\tau+h^{4}\right)$. Finally, a numerical example is given to demonstrate the correctness of the theoretical analysis and the validity of the scheme.

The rest of the paper is arranged as follows. In Section 2, we give the semidiscrete finite element scheme and fully discrete finite element scheme for problem (1) and conduct convergence analysis. A numerical example is given in Section 3. In section 4, we present conclusions and some future works.

\section{Finite Element Approximation}

When finite element method is used to solve this kind of equation, time variable $t$ is usually treated as parameter. Let $I=[0, L]$, introducing the Sobolev space $H_{0}^{2}(I)=\left\{u \mid u \in H^{2}(I), u(0, t)=u(L, t)=0, u_{x}(0, t)=u_{x}(L, t)=0\right\}$. For any $v(x) \in H_{0}^{2}(I)$ and for fixed $t$, multiply both sides of equation (1) by $v(x)$ and integrate, we have

$$
\int_{0}^{L}\left[E I \frac{\partial^{4} u}{\partial x^{4}}+\rho_{0} S \frac{\partial^{2} u}{\partial t^{2}}+\mu \frac{\partial u}{\partial t}+k u\right] v(x) \mathrm{d} x=\int_{0}^{L} f v(x) \mathrm{d} x .
$$

Integrate by parts with respect to $\frac{\partial^{4} u}{\partial x^{4}} v(x)$ and pay attention to the boundary 
conditions and the value of $v(x)$. Based on (2), we can obtain the weak formulation of (1): find $u \in H_{0}^{2}$, satisfying

$$
\left\{\begin{array}{l}
\left(E I u_{x x}, v_{x x}\right)+\left(\rho_{0} S u_{t t}, v\right)+\left(\mu u_{t}, v\right)+(k u, v)=(f, v), \forall v \in H_{0}^{2}, \\
u(x, 0)=\varphi(x), u_{t}(x, 0)=\psi(x) .
\end{array}\right.
$$

\subsection{Error Estimation of Semi-Discrete Finite Element Schemes}

Let $I_{h}: 0=x_{0}<x_{1}<\cdots<x_{M}=L$ be an uniform partition of the interval $[0, L]$, $h=\frac{L}{M}, x_{j}=j h, j=0,1,2, \cdots, M$. Let $V_{h}$ be a finite-dimensional subspace of $H_{0}^{2}(I)$ constituted by piecewise cubic Hermite type polynomials on $I_{h}$. We can define the semi-discrete Galerkin finite element approximation of (4): find $u_{h} \in V_{h}$ satisfying

$$
\left(E I u_{h, x x}, v_{h, x x}\right)+\left(\rho_{0} S u_{h, t t}, v_{h}\right)+\left(\mu u_{h, t}, v_{h}\right)+\left(k u_{h}, v_{h}\right)=\left(f, v_{h}\right), \forall v_{h} \in V_{h} .
$$

In order to estimate the error, we first define the elliptic projection $R_{h}: H_{0}^{2}(I) \rightarrow V_{h}$ such that

$$
\left(E I u_{x x}, \chi_{x x}\right)+(k u, \chi)=\left(E I R_{h} u_{x x}, \chi_{x x}\right)+\left(k R_{h} u, \chi\right), \forall \chi \in V_{h} .
$$

A well known error estimate (see [11]) is the following.

Lemma 2.1. $1 \leq k \leq 3$ denotes the degree of finite element space $V_{h}$, for any $u \in H_{0}^{2} \cap H^{k+1}$, we have

$$
\left\|u-R_{h} u\right\|+h\left\|u-R_{h} u\right\|_{1}+h^{2}\left\|u-R_{h} u\right\|_{2} \leq C h^{k+1}\|u\|_{k+1} .
$$

Now, we prove the estimate for the error between the solutions of the semidiscrete and continuous problems. Denote $\rho=u-R_{h} u$ and $\theta=R_{h} u-u_{h}$, the first important conclusion of this paper is as follows.

Theorem 2.1. Let $u$ and $u_{h}$ be the solutions of (1) and (4), respectively. $u \in H_{0}^{2} \cap H^{4}$. Then

$$
\left\|u-u_{h}\right\| \leq C h^{4}\left[\|u\|_{4}+\left(\int_{0}^{t}\left\|u_{t t}\right\|_{4}^{2}+\left\|u_{t}\right\|_{4}^{2} \mathrm{~d} s\right)^{\frac{1}{2}}\right],
$$

where $C$ is a constant, independent of $L$ and $h$.

Proof. We can obtain easily by Lemma 2.1 that

$$
\|\rho\|=\left\|u-R_{h} u\right\| \leq C h^{4}\|u\|_{4} .
$$

Combining (3) and (4), we get the following error equation

$$
\begin{aligned}
& \left(E I \theta_{x x}, v_{h, x x}\right)+\left(\rho_{0} S \theta_{t t}, v_{h}\right)+\left(\mu \theta_{t}, v_{h}\right)+\left(k \theta, v_{h}\right) \\
& =-\left(\rho_{0} S \rho_{t t}, v_{h}\right)-\left(\mu \rho_{t}, v_{h}\right) .
\end{aligned}
$$

$\theta_{t}$ belongs to $V_{h}$. Thus, choosing $v_{h}=\theta_{t}$ in (9), we conclude that

$$
\begin{aligned}
& \left(E I \theta_{x x}, \theta_{t, x x}\right)+\left(\rho_{0} S \theta_{t t}, \theta_{t}\right)+\left(\mu \theta_{t}, \theta_{t}\right)+\left(k \theta, \theta_{t}\right) \\
& =-\left(\rho_{0} S \rho_{t t}, \theta_{t}\right)-\left(\mu \rho_{t}, \theta_{t}\right) .
\end{aligned}
$$

Using $\mathcal{E}$-Cauchy inequality gives 


$$
\begin{aligned}
& \frac{E I}{2} \frac{\mathrm{d}}{\mathrm{d} t}\left\|\theta_{x x}\right\|^{2}+\frac{\rho_{0} S}{2} \frac{\mathrm{d}}{\mathrm{d} t}\left\|\theta_{t}\right\|^{2}+\mu\left\|\theta_{t}\right\|^{2}+\frac{k}{2} \frac{\mathrm{d}}{\mathrm{d} t}\|\theta\|^{2} \\
& \leq \frac{\rho_{0}^{2} S^{2}}{2 \mu}\left\|\rho_{t t}\right\|^{2}+\frac{\mu}{2}\left\|\theta_{t}\right\|^{2}+\frac{\mu}{2}\left\|\rho_{t}\right\|^{2}+\frac{\mu}{2}\left\|\theta_{t}\right\|^{2} .
\end{aligned}
$$

Integrate the above inequality over $[0, t]$

$$
\begin{aligned}
& E I\left\|\theta_{x x}\right\|^{2}+\rho_{0} S\left\|\theta_{t}\right\|^{2}+k\|\theta\|^{2} \\
& \leq E I\left\|\theta_{x x}(0)\right\|^{2}+\rho_{0} S\left\|\theta_{t}(0)\right\|^{2}+\int_{0}^{t} \frac{\rho_{0}^{2} S^{2}}{\mu}\left\|\rho_{t t}\right\|^{2} \mathrm{~d} s+\int_{0}^{t} \mu\left\|\rho_{t}\right\|^{2} \mathrm{~d} s .
\end{aligned}
$$

Here the first and the second are nonnegative, setting $\left\|\theta_{x x}(0)\right\|=0,\left\|\theta_{t}(0)\right\|=0$ yields

$$
\|\theta\|^{2} \leq C h^{8} \int_{0}^{t}\left\|u_{t t}\right\|_{4}^{2}+\left\|u_{t}\right\|_{4}^{2} \mathrm{ds}
$$

It follows as above that

$$
\|\theta\| \leq C h^{4}\left(\int_{0}^{t}\left\|u_{t t}\right\|_{4}^{2}+\left\|u_{t}\right\|_{4}^{2} \mathrm{~d} s\right)^{\frac{1}{2}} .
$$

By the triangle inequality, we deduce that

$$
\left\|u-u_{h}\right\|=\left\|u-R_{h} u+R_{h} u-u_{h}\right\| \leq\|\rho\|+\|\theta\| .
$$

Combing (8) and (13), we complete the proof.

\subsection{Error Estimation of Fully Discrete Finite Element Schemes}

Let $0=t_{0}<t_{1}<\cdots<t_{N}=T$ be an uniform partition of the interval $[0, T]$, $\tau=\frac{T}{N}, t_{n}=n \tau, n=0,1,2, \cdots, N$. Replacing the first and the second time derivative in equation (4) by backward difference quotient and central difference quotient respectively, full-discrete finite element form of problem (1) can be define: find $u_{h}^{n} \in V_{h}$ such that

$$
\begin{aligned}
& \left(E I u_{h, x x}^{n+1}, v_{h, x x}\right)+\left(\rho_{0} S \frac{u_{h}^{n+1}-2 u_{h}^{n}+u_{h}^{n-1}}{\tau^{2}}, v_{h}\right)+\left(\mu \frac{u_{h}^{n+1}-u_{h}^{n}}{\tau}, v_{h}\right) \\
& +\left(k u_{h}^{n+1}, v_{h}\right)=\left(f^{n+1}, v_{h}\right), \forall v_{h} \in V_{h} .
\end{aligned}
$$

Denote $\rho^{n+1}=u^{n+1}-R_{h} u^{n+1}$ and $\theta^{n+1}=R_{h} u^{n+1}-u_{h}^{n+1}$ in analogy with definitions before. We obtain theorem 2.2 which estimates the error between the solutions of the fully discrete and continuous problems.

Theorem 2.2. Let $u$ and $u_{h}$ be the solutions of (1) and(15), respectively. $u \in H_{0}^{2} \cap H^{4}$. Then

$$
\left\|u^{n+1}-u_{h}^{n+1}\right\| \leq C\left(\tau+h^{4}\right),
$$

where $C$ is a constant, independent of $T$ and $\tau$.

Proof. Minusing (3) and (15), we get

$$
\left(E I \theta_{x x}^{n+1}, v_{h, x x}\right)+\left(\rho_{0} S \frac{\theta^{n+1}-2 \theta^{n}+\theta^{n-1}}{\tau^{2}}, v_{h}\right)+\left(\mu \frac{\theta^{n+1}-\theta^{n}}{\tau}, v_{h}\right)+\left(k \theta^{n+1}, v_{h}\right)
$$




$$
\begin{aligned}
& =\left(\rho_{0} S\left(\frac{u^{n+1}-2 u^{n}+u^{n-1}}{\tau^{2}}-u_{t t}^{n+1}\right), v_{h}\right)-\left(\rho_{0} S \frac{\rho^{n+1}-2 \rho^{n}+\rho^{n-1}}{\tau^{2}}, v_{h}\right) \\
& +\left(\mu\left(\frac{u^{n+1}-u^{n}}{\tau}-u_{t}^{n+1}\right), v_{h}\right)-\left(\mu \frac{\rho^{n+1}-\rho^{n}}{\tau}, v_{h}\right) .
\end{aligned}
$$

Setting $v_{h}=\frac{\theta^{n+1}-\theta^{n}}{\tau}$ in (17) and using $\mathcal{E}$-Cauchy inequality, we observe that

$$
\begin{aligned}
& \frac{E I}{2 \tau}\left(\left\|\theta_{x x}^{n+1}\right\|^{2}-\left\|\theta_{x x}^{n}\right\|^{2}\right)+\frac{\rho_{0} S}{2 \tau}\left(\left\|\frac{\theta^{n+1}-\theta^{n}}{\tau}\right\|^{2}-\left\|\frac{\theta^{n}-\theta^{n-1}}{\tau}\right\|^{2}\right)+\frac{k}{2 \tau}\left(\left\|\theta^{n+1}\right\|^{2}-\left\|\theta^{n}\right\|^{2}\right) \\
& \leq \frac{\rho_{0}^{2} S^{2}}{\mu}\left\|\frac{u^{n+1}-2 u^{n}+u^{n-1}}{\tau^{2}}-u_{t}^{n+1}\right\|^{2}+\frac{\rho_{0}^{2} S^{2}}{\mu}\left\|\frac{\rho^{n+1}-2 \rho^{n}+\rho^{n-1}}{\tau^{2}}\right\|^{2} \\
& +\mu\left\|\frac{u^{n+1}-u^{n}}{\tau}-u_{t}^{n+1}\right\|^{2}+\mu\left\|\frac{\rho^{n+1}-\rho^{n}}{\tau}\right\|^{2} .
\end{aligned}
$$

Next, we will estimate the right end of equation (18) one by one. By using Taylor expansion, we deduce

$$
\begin{gathered}
\left|\frac{u^{n+1}-2 u^{n}+u^{n-1}}{\tau^{2}}-u_{t t}^{n+1}\right|^{2} \leq \frac{33 \tau}{10} \int_{t_{n-1}}^{t_{n+1}}\left|\frac{\partial^{3} u}{\partial t^{3}}\right|^{2} \mathrm{~d} s . \\
\left|\frac{\rho^{n+1}-2 \rho^{n}+\rho^{n-1}}{\tau^{2}}\right|^{2}=\left|\frac{1}{\tau^{2}}\left[\int_{t_{n}}^{t_{n+1}} \rho_{t t}(s)\left(t_{n+1}-s\right) \mathrm{d} s+\int_{t_{n-1}}^{t_{n}} \rho_{t t}(s)\left(s-t_{n-1}\right) \mathrm{d} s\right]\right|^{2} . \\
\left|\frac{u^{n+1}-u^{n}}{\tau}-u_{t}^{n+1}\right|^{2}=\left|\frac{1}{\tau} \int_{t_{n}}^{t_{n+1}} u_{t t}(s)\left(t_{n}-s\right) \mathrm{d} s\right|^{2} . \\
\left|\frac{\rho^{n+1}-\rho^{n}}{\tau}\right|^{2}=\left|\frac{1}{\tau} \int_{t_{n}}^{t_{n+1}} \rho_{t}(s) \mathrm{d} s\right|^{2} .
\end{gathered}
$$

Therefore, from (19), it is immediate to realize that

$$
\left\|\frac{u^{n+1}-2 u^{n}+u^{n-1}}{\tau^{2}}-u_{t t}^{n+1}\right\|^{2} \leq \frac{33 \tau}{10} \int_{t_{n-1}}^{t_{n+1}}\left\|\frac{\partial^{3} u}{\partial t^{3}}\right\|^{2} \text { ds. }
$$

Using Schwarz inequality we can verify

$$
\left\|\int_{a}^{b} f(x, t) \mathrm{d} t\right\|^{2} \leq(b-a) \int_{a}^{b}\|f\|^{2} \mathrm{~d} t .
$$

Then, we observe that (20), (24) and Lemma 2.1 yield

$$
\begin{aligned}
& \left\|\frac{\rho^{n+1}-2 \rho^{n}+\rho^{n-1}}{\tau^{2}}\right\|^{2} \\
& \leq\left\|\frac{1}{\tau^{2}} \int_{t_{n}}^{t_{n+1}} \rho_{t t}(s)\left(t_{n+1}-s\right) \mathrm{d} s\right\|^{2}+\left\|\frac{1}{\tau^{2}} \int_{t_{n-1}}^{t_{n}} \rho_{t t}(s)\left(s-t_{n-1}\right) \mathrm{d} s\right\|^{2} \\
& \leq \frac{1}{\tau} \int_{t_{n}}^{t_{n+1}}\left\|\rho_{t t}(s)\right\|^{2} \mathrm{~d} s+\frac{1}{\tau} \int_{t_{n-1}}^{t_{n}}\left\|\rho_{t t}(s)\right\|^{2} \mathrm{~d} s \\
& =\frac{1}{\tau} \int_{t_{n-1}}^{t_{n+1}}\left\|\rho_{t t}(s)\right\|^{2} \mathrm{~d} s \leq \frac{1}{\tau} C h^{8} \int_{t_{n-1}}^{t_{n+1}}\left\|u_{t t}\right\|_{4}^{2} \mathrm{~d} s .
\end{aligned}
$$


Combining (21) and (24) and Lemma 2.1, we have

$$
\left\|\frac{u^{n+1}-u^{n}}{\tau}-u_{t}^{n+1}\right\|^{2}=\left\|\frac{1}{\tau} \int_{t_{n}}^{t_{n+1}} u_{t t}(s)\left(t_{n}-s\right)\right\|^{2} \leq \tau \int_{t_{n}}^{t_{n+1}}\left\|u_{t t}(s)\right\|^{2} \mathrm{~d} s .
$$

Analogously, combining (22), (24) and Lemma 2.1, we deduce

$$
\left\|\frac{\rho^{n+1}-\rho^{n}}{\tau}\right\|^{2}=\left\|\frac{1}{\tau} \int_{t_{n}}^{t_{n+1}} \rho_{t}(s) \mathrm{d} s\right\|^{2} \leq \frac{1}{\tau} \int_{t_{n}}^{t_{n+1}}\left\|\rho_{t}(s)\right\|^{2} \mathrm{~d} s \leq \frac{1}{\tau} C h^{8} \int_{t_{n}}^{t_{n+1}}\left\|u_{t}\right\|_{4}^{2} \mathrm{~d} s .
$$

Now we combine (23), (25), (26) and (27) to have

$$
\begin{aligned}
& \frac{E I}{2 \tau}\left(\left\|\theta_{x x}^{n+1}\right\|^{2}-\left\|\theta_{x x}^{n}\right\|^{2}\right)+\frac{\rho_{0} S}{2 \tau}\left(\left\|\frac{\theta^{n+1}-\theta^{n}}{\tau}\right\|^{2}-\left\|\frac{\theta^{n}-\theta^{n-1}}{\tau}\right\|^{2}\right)+\frac{k}{2 \tau}\left(\left\|\theta^{n+1}\right\|^{2}-\left\|\theta^{n}\right\|^{2}\right) \\
& \leq \frac{33}{10} \frac{\rho_{0}^{2} S^{2}}{\mu} \tau \int_{t_{n-1}}^{t_{n+1}}\left\|\frac{\partial^{3} u}{\partial t^{3}}\right\|^{2} \mathrm{~d} s+\frac{1}{\tau} \frac{\rho_{0}^{2} S^{2}}{\mu} C h^{8} \int_{t_{n-1}}^{t_{n+1}}\left\|u_{t t}\right\|_{4}^{2} \mathrm{~d} s \\
& \quad+\tau \mu \int_{t_{n}}^{t_{n+1}}\left\|u_{t t}\right\|^{2} \mathrm{~d} s+\frac{\mu}{\tau} C h^{8} \int_{t_{n}}^{t_{n+1}}\left\|u_{t}\right\|_{4}^{2} \mathrm{~d} s .
\end{aligned}
$$

Multiplying equation (28) by $\tau$ and taking the sum of $n$, we derive

$$
\begin{aligned}
& \frac{E I}{2}\left\|\theta_{x x}^{n+1}\right\|^{2}+\frac{\rho_{0} S}{2}\left\|\frac{\theta^{n+1}-\theta^{n}}{\tau}\right\|^{2}+\frac{k}{2}\left\|\theta^{n+1}\right\|^{2} \\
& \leq \frac{E I}{2}\left\|\theta_{x x}^{1}\right\|^{2}+\frac{\rho_{0} S}{2}\left\|\frac{\theta^{1}-\theta^{0}}{\tau}\right\|^{2}+\frac{k}{2}\left\|\theta^{1}\right\|^{2}+\frac{33}{10} \frac{\rho_{0}^{2} S^{2}}{\mu} \tau^{2} \int_{0}^{t_{n+1}}\left\|\frac{\partial^{3} u}{\partial t^{3}}\right\|^{2} \mathrm{~d} s \\
& +\frac{\rho_{0}^{2} S^{2}}{\mu} C h^{8} \int_{0}^{t_{n+1}}\left\|u_{t t}\right\|_{4}^{2} \mathrm{~d} s+\tau^{2} \mu \int_{t_{1}}^{t_{n+1}}\left\|u_{t t}\right\|^{2} \mathrm{~d} s+\mu C h^{8} \int_{t_{1}}^{t_{n+1}}\left\|u_{t}\right\|_{4}^{2} \mathrm{~d} s .
\end{aligned}
$$

That is

$$
\left\|\theta^{n+1}\right\| \leq C\left(\tau+h^{4}\right) .
$$

On the other hand, by Lemma 2.1, we have

$$
\left\|\rho^{n+1}\right\| \leq C h^{4} .
$$

Using triangle inequality and the above estimates gives the proof.

\section{Numerical Experiment}

In order to prove the validity of the theoretical analysis, we give the problem for which the exact solution is given. We apply the full-discrete scheme proposed above to solve this problem.

Example. Let the variables $E I, \rho_{0}, S, \mu, k, L$ and $T$ in problem (1) be equal to 1. Consider the following initial-boundary value problem:

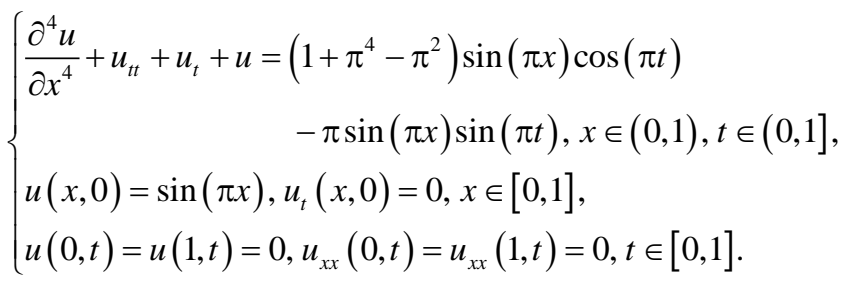

The exact solution is $u(x, t)=\sin (\pi x) \cos (\pi t)$. 
Table 1. Errors and convergence order of Example.

\begin{tabular}{cccc}
\hline$h$ & $\tau$ & $L^{2}$-error & order \\
\hline$\frac{1}{2}$ & $\frac{1}{2^{4}}$ & 0.00252081 & - \\
$\frac{1}{2^{2}}$ & $\frac{1}{2^{8}}$ & 0.0001626 & 3.9545 \\
$\frac{1}{2^{3}}$ & $\frac{1}{2^{12}}$ & $1.02427 \mathrm{e}-05$ & 3.9887 \\
$\frac{1}{2^{4}}$ & $\frac{1}{2^{16}}$ & $6.41423 \mathrm{e}-07$ & 3.9972 \\
$\frac{1}{2^{5}}$ & $\frac{1}{2^{20}}$ & $4.01086 \mathrm{e}-08$ & 3.9993 \\
\hline
\end{tabular}

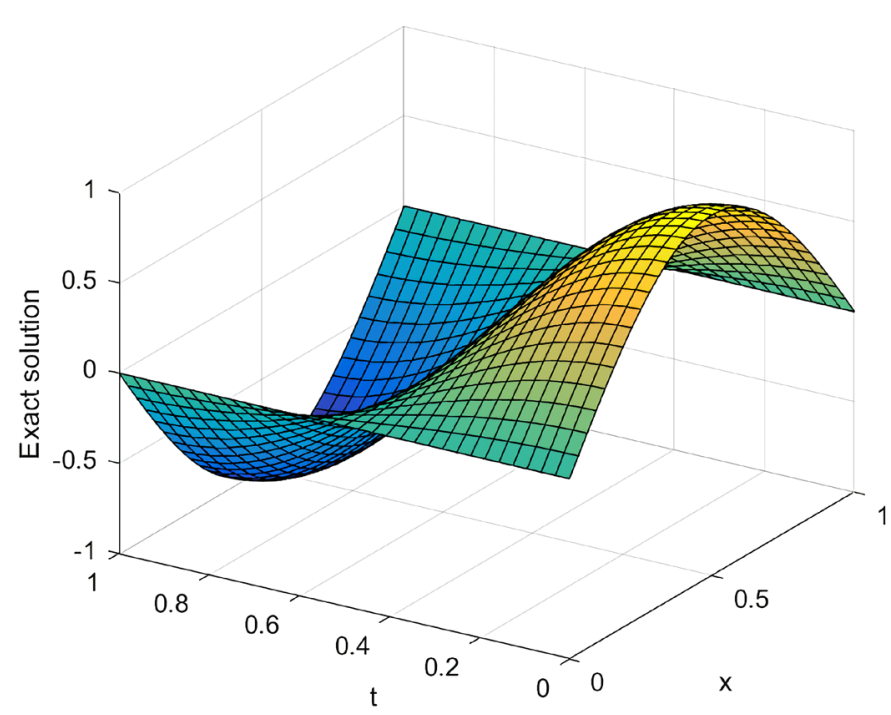

Figure 1. Exact solution with $h=\frac{1}{2^{5}}$.

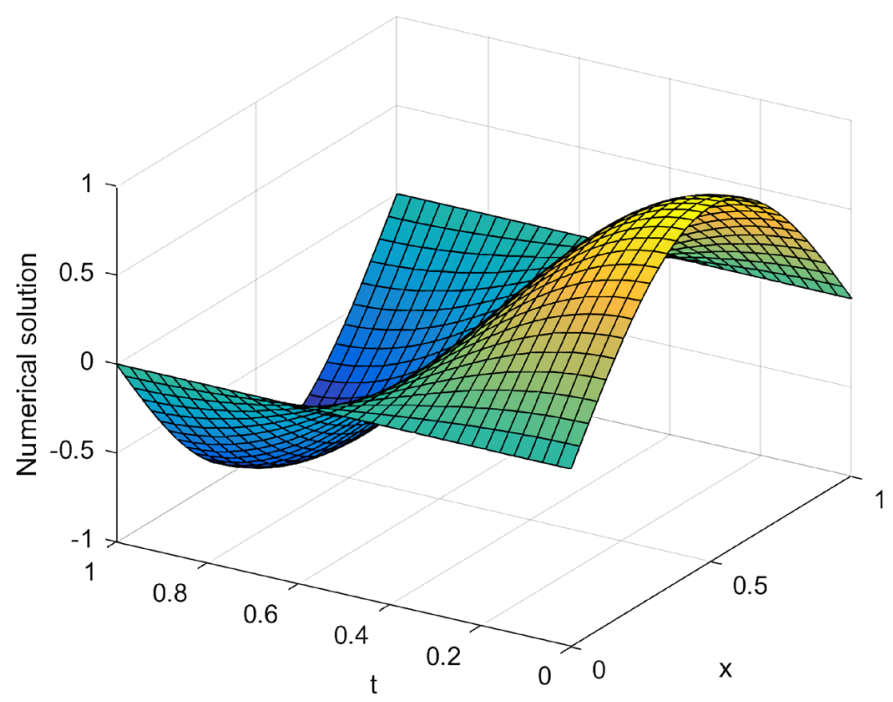

Figure 2. Solution of fully discrete finite element schemes with $h=\frac{1}{2^{5}}$. 
We use the finite element fully discrete scheme to solve the problem. In Table 1 , by choosing $\tau=h^{4}$, we give the $L^{2}$-error and the order of spatial convergence. The results show that the space convergence order of the scheme is fourth order. Surface plots of the exact solution and the numerical solution are presented in Figure 1, Figure 2.

\section{Conclusions}

Based on Hermite finite element method, the vibration of viscoelastic beam is analyzed numerically. Semi-discrete and fully discrete Hermite finite element formats are given. By means of a numerical example, the $L^{2}$-error and the convergence order between the exact solution of the original equation and the finite element solution are given. The validity of the proposed scheme in theoretical applications is verified.

Since the equations in this paper are one-dimensional in space, high-order finite element method such as finite element method for two-dimensional plate vibration problem will be the direction of our future research.

\section{Funding}

The work is supported by National Natural Science Foundation, China (11501335).

\section{Acknowledgements}

The authors would like to thank editor and referees for their valuable advice for the improvement of this article.

\section{Conflicts of Interest}

The authors declare no conflicts of interest regarding the publication of this paper.

\section{References}

[1] Putrevu, H., Subramanian, H. and Mulay, S.S. (2021) On the Viscoelastic Dynamic Beam Modelling. International Journal of Advances in Engineering Sciences and Applied Mathematics, 13, 18-32. https://doi.org/10.1007/s12572-021-00288-8

[2] Pierro, E. (2020) Damping Control in Viscoelastic Beam Dynamics. Journal of Vibration and Control, 0, 1-12. https://doi.org/10.1177/1077546320903195

[3] Huang, Z.C., Wang, X.G., Wu, N.X., Chu, F.L. and Luo, J. (2019) A Finite Element Model for the Vibration Analysis of Sandwich Beam with Frequency-Dependent Viscoelastic Material Core. Materials, 12, No. 20.

[4] Kouami, K., Foudil, M., Erasmo, C. and Mostafa, D.E. (2021) A Finite Element Approach for the Static and Vibration Analyses of Functionally Graded Material Viscoelastic Sandwich Beams with Nonlinear Material Behavior. Composite Structures, 274, Article ID: 114315.

[5] Snchez, E.D., Nallim, L.G., Bellomo, F.J. and Oller, S.H. (2019) Generalized Viscoelastic Model for Laminated Beams Using Hierarchical Finite Elements. Composite Structures, 235, Article ID: 111794. https://doi.org/10.1016/j.compstruct.2019.111794 
[6] Lekdim, B. and Khemmoudj, A. (2021) Existence and Energy Decay of Solution to a Nonlinear Viscoelastic Two-Dimensional Beam with a Delay. Multidimensional Systems and Signal Processing, 32, 915-931.

[7] Wu, Q.L. and Qi, G.Y. (2020) Viscoelastic String-Beam Coupled Vibro-Impact System: Modeling and Dynamic Analysis. European Journal of Mechanics-A/Solids, 82, Article ID: 104012. https://doi.org/10.1016/j.euromechsol.2020.104012

[8] Elhuni, H. and Basu, D. (2019) Dynamic Soil Structure Interaction Model for Beams on Viscoelastic Foundations Subjected to Oscillatory and Moving Loads. Computers and Geotechnics, 115, Article ID: 103157.

https://doi.org/10.1016/j.compgeo.2019.103157

[9] Yu, C., Zhang, J., Chen, Y., Feng, Y.J. and Yang, A.M. (2019) A Numerical Method for Solving Fractional-Order Viscoelastic Euler-Bernoulli Beams. Chaos, Solitons \& Fractals, 128, 275-279. https://doi.org/10.1016/j.chaos.2019.07.035

[10] Li, R.H. and Liu, B. (2009) Numerical Solution of Differential Equation. Higher Education Press, Beijing.

[11] Ciarlet, P. (1978) The Finite Element Method for Elliptic Problems. North-Holland, Amsterdam. 